\title{
$\angle S$ Research Square \\ Parenteral nutrition with fish oil-based lipid emulsion reduces the risk of cholestasis in preterm infants
}

\section{YI-LING WANG}

Changhua Christian Children's Hospital https://orcid.org/0000-0003-2948-4347

\section{Lih-Ju Chen}

Changhua Christian Children's Hospital

Lon-Yen Tsao

Changhua Christian Children's Hospital

Hsiao-Neng Chen

Changhua Christian Children's Hospital

Cheng-Han Lee

Changhua Christian Children's Hospital

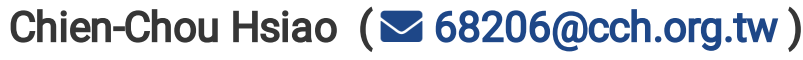

\section{Research article}

Keywords: preterm infant, fish oil, parenteral nutrition, cholestasis, DHA

Posted Date: September 17th, 2019

DOI: https://doi.org/10.21203/rs.2.14547/v1

License: (c) (i) This work is licensed under a Creative Commons Attribution 4.0 International License. Read Full License 


\section{Abstract}

Background Preterm infants received long-term parenteral nutrition (PN) due to gastrointestinal immaturity. Mixed lipid emulsions composed of soybean oil, medium chain triglycerides (MCTs), olive oil, and fish oil, which have a relatively low $\omega-6$ : $\omega-3$ ratio, may decrease the incidence of PN-associated cholestasis by reducing oxidative stress and providing an anti-inflammatory effect.

Methods The retrospective cohort study enrolled a total 399 very low birth weight (VLBW) premature infants between January 2009 and November 2017 at a single neonatal intensive care unit. Preterm infants received total parenteral nutrition with either mixed lipid emulsion (SMOFlipid, $n=195$ ) or soybean oil-based lipid emulsion (Lipovenoes, $n=204$ ) for at least seven days. We compared the outcomes of PN-associated cholestasis, co-morbidities and mortality.

Results The incidence of PN-associated cholestasis was significantly lower in the SMOFlipid group than the Lipovenoes group $(10.3 \%$ vs $20.1 \%, P=0.006)$. The related clinical laboratory findings, including levels of $y-G T(P=0.019)$, triglyceride $(P<0.001)$, and cholesterol $(P=0.023)$, were significantly lower in the SMOFlipid group. The duration to full feeding days shortened in the SMOFlipid group compared with the Lipovenoes group ( $25 \pm 10.33$ vs $33 \pm 16.22$, $\mathrm{P}<0.001)$. Relevant complications, such as severe retinopathy of prematurity (ROP, $3.6 \%$ vs $14.3 \%, \mathrm{P}<0.001$ ) and bronchopulmonary dysplasia (BPD, $36.9 \%$ vs $46.7 \%, \mathrm{P}=0.046$ ) were also reduced in the SMOFlipid group versus the Lipovenoes group, but there was no significant effect on severe intraventricular hemorrhage (IVH), necrotizing enterocolitis (NEC), or mortality in both groups.

Conclusions In very premature infants, $\mathrm{PN}$ with fish oil-based lipid emulsions is associated with a lower incidence of PN-associated cholestasis compared with soybean oil-based lipid emulsions.

\section{Introduction}

Preterm neonates rely on parenteral nutrition (PN) for several weeks after birth. Early administration of milk feeding is insufficient to support growth owing to gastrointestinal immaturity. However, the risk of infection, hypertriglyceridemia, hyperbilirubinemia, and $\mathrm{PN}$-associated liver disease increase in the infants receiving long-term PN [1-2]. There is a growing amount of evidence that soybean oil emulsions containing a high proportion of $\omega-6$ long-chain polyunsaturated fatty acids (LC-PUFAs) and phytosterols induce immune function and promote hepatic damage [1-5]. The latest generation of mixed lipid emulsions are composed of soybean, medium-chain triglycerides (MCTs), olive oil, and fish oil (SMOFlipid). Soybean oil provides essential fatty acids, such as linoleic acid (LA) and a-linolenic acid (aLNA) [2-3]. MCTs are more beneficial for faster metabolic clearance than long-chain triglycerides (LCTs) [6-8]. Olive oil is rich in monounsaturated fatty acids (MUFAs), which protect against lipid peroxidation $[4,9,10]$. Fish oil provides $\omega-3$ LC-PUFAs, eicosapentaenoic acid (EPA), and docosahexaenoic acid (DHA), which reduce oxidative stress, suppress inflammation, and prevent PN-associated cholestasis [2$5,11]$. A balanced fatty acid supply influences outcomes such as growth, visual development and 
cognitive development [12-14]. Therefore, we hypothesized that SMOFlipid may be associated with decreasing the incidence of $\mathrm{PN}$-associated cholestasis compared with soybean oil-based lipid emulsion in preterm infants.

\section{Methods}

This retrospective cohort study was performed at the Department of Neonatology, Changhua Christian Children Hospital, Taiwan, between January 2009 and November 2017. A total of 399 premature neonates who were eligible, of gestational age $\leq 32$ weeks, birth weight $\leq 1,250 \mathrm{~g}$, and received parenteral nutrition for at least seven days, were enrolled. The study was approved by the Institutional Review Board at Changhua Christian Hospital. Infants with conditions associated with cholestasis independent of PN (i.e., infection with cytomegalovirus, HIV, hepatitis B or C), hemolytic diseases, primary liver diseases, inborn errors of metabolism, multiorgan failure or hypoxic-ischemic encephalopathy were excluded. The first cohort of 204 preterm infants from January 2009 to April 2013 received soybean oil-based lipid emulsion (Lipovenoes 20\%, control group). The second cohort of 195 preterm infants from May 2013 to November 2017 received SMOFlipid 20\% (study group) composed of $30 \%$ soybean oil, $30 \%$ MCTs, $25 \%$ olive oil, and $15 \%$ fish oil as the PN lipid emulsion. Both cohorts were cared for by the same multidisciplinary team, including medical, pharmacy, dietetic, nursing, and nutritional care.

Regardless of whether the PN contained SMOFlipid or Lipovenoes, the dosage of both groups started at $1 \mathrm{~g} / \mathrm{kg} /$ day on day 1 , and this was increased daily by increments of $1 \mathrm{~g} / \mathrm{kg} /$ day up to a maximum of $3 \mathrm{~g} / \mathrm{kg} /$ day. The lipid emulsion was administered as part of a PN regimen along with other nutrients (glucose, amino acids, vitamins, trace elements, and electrolytes) in two separate syringes. The dosages of other nutrients were standardized in accordance with current recommendations [1]. The infants received nasogastric tube feeding supplement with formula or breast milk as early as possible. All preterm infants received probiotics after they started feeding. PN was withdrawn when infants received enteral energy $>80 \mathrm{kcal} / \mathrm{kg} /$ day.

Demographic data, medical history, concomitant diseases, clinical assessments, and medications of the infants were recorded daily beginning on day 0 . Blood samples for assessment of parameters for safety and efficacy evaluation were obtained weekly. Adverse events and their possible relation to study treatment were documented. The primary outcome, $\mathrm{PN}$-associated cholestasis, was defined as serum direct bilirubin levels more than $1.5 \mathrm{mg} / \mathrm{dl}$, and greater than $20 \%$ of serum total bilirubin levels. Peak levels of liver enzymes (aspartate aminotransferase [GOT], alanine aminotransferase [GPT], $ү$-glutamyl transferase [yGT], alkaline phosphatase [ALP]), c-reactive protein (CRP), albumin, cholesterol, and triglyceride, were also analyzed during hospitalization. Secondary clinical and developmental outcomes included severe retinopathy of prematurity (ROP), intraventricular hemorrhage (IVH), necrotizing enterocolitis (NEC), bronchopulmonary dysplasia (BPD), sepsis and mortality. ROP was screened by an ophthalmologist beginning at four weeks of age. Treatment with intravitreal bevacizumab was performed at ROP $\geq$ stage 3 . IVH was diagnosed by cerebral ultrasound on day 0, 3, 7, 21 and monthly. Severe IVH was identified as stage $\geq 3$. NEC was diagnosed clinically (Bell's stage $\geq$ lla) or after surgical exploration. 
BPD was defined as requiring an oxygen supplement after 36 weeks postmenstrual age. Sepsis was proven by blood culture.

Data from all of the assessments were tabulated using descriptive statistics. Standard summary statistics and $95 \%$ confidence intervals were calculated appropriately. To compare the mean values from parametric variables, data were analyzed using the Student $t$ test. Secondary outcomes were compared between the groups with the $\chi^{2}$ or Fisher's exact tests when appropriate for categorical data. A $P$ Value of $<0.05$ was considered statistically significant. Statistical evaluation of results was carried out for the entire patient population and according to the stratification. The results of the study and control groups are presented below.

\section{Results}

A total of 399 preterm infants were enrolled: 195 in the SMOFlipid group and 204 in the Lipovenoes group. In the baseline characteristics (Table 1), there were no significant differences between the SMOFlipid and Lipovenoes groups, with regard to gestational age ( 28.9 weeks \pm 2.5 vs 28.8 weeks \pm 2.5 ), birth weight $(1,122.9 \mathrm{~g} \pm 294.6$ vs $1,111.5 \mathrm{~g} \pm 286.9)$, height $(36.5 \mathrm{~cm} \pm 3.8$ vs $36.4 \mathrm{~cm} \pm 3.5)$, head circumference $(26.2 \mathrm{~cm} \pm 2.8$ vs $25.8 \mathrm{~cm} \pm 2.5)$, and the Apgar score at one minute $(6.2 \pm 1.7 \mathrm{vs} 6.0 \pm 1.6)$ and at five minutes $(8.0 \pm 1.2$ vs $7.8 \pm 1.3)$. Proportion of breast milk feeding was similar in the SMOFlipid group and the Lipovenoes group $(55.9 \%$ vs $62 \%, P=0.083)$. However, the average parenteral nutrition days $(18 \pm 9.31$ vs $25.5 \pm 14.36, P<0.001)$ and duration to full feeding days $(25 \pm 10.33$ vs $33 \pm 16.22, P$ $<0.001)$ reduced in the SMOFlipid group compared with Lipovenoes group.

The primary outcome was that $\mathrm{PN}$-associated cholestasis significantly decreased in the SMOFlipid group compared with the Lipovenoes group $(10.3 \%$ vs $20.1 \%, P=0.006)$ [Table 2]. Multivariable logistic regression results showed that a higher GPT level (OR 1.012; 95\% Cl: 1.001-1.022, $P=0.024$ ), lower Hct level (OR 0.964; 95\% Cl: 0.947-0.982, $P<0.001$ ), hypoalbuminemia (OR 0.353; 95\% Cl: 0.163-0.767, $P=$ 0.008 ), and hypoglycemia (OR $0.973 ; 95 \% \mathrm{Cl}: 0.954-0.992, P=0.005$ ) were significantly correlative to cholestasis (Table 3). The relevant clinical laboratory results also showed significant decreases in the SMOFlipid group, including $Y$ - GT (107.6 \pm 92.7 vs $131.1 \pm 98.8, P=0.019)$, triglyceride $(127.9 \pm 70.1$ vs $186.8 \pm 165.3, P<0.001)$, and cholesterol $(150.4 \pm 33.9$ vs $158.4 \pm 35.9, P=0.023)$ levels (Table 2$)$.

During the treatment period, the c-reactive protein level, white blood-cell count, Hemoglobin, platelet count and the rate of sepsis were no significant difference between the SMOFlipid group and the Lipovenoes group. Other secondary outcomes (Table 4) showed ROP significantly decreased in the SMOFlipid group (3.6\% vs $14.3 \%, P<0.001)$. The incidence of BPD was slightly lower in the SMOFlipid group ( $36.9 \%$ vs $46.7 \%, P=0.046)$, but there was no difference in severe IVH ( $4.1 \%$ vs $2.5 \%, P=0.235)$, NEC ( $5.6 \%$ vs $7.4 \%$, $P=0.489)$ or mortality $(1.0 \%$ vs $3.4 \%, P=0.176)$ in both groups.

\section{Discussion}


PN-associated cholestasis is the most concerned morbidity in preterm infants with long-term use of parenteral nutrition. In this retrospective cohort study between January 2009 and November 2017, a mixed lipid emulsion composed of soybean oil, MCT, olive oil, and fish oil significantly reduced the incidence of PN-associated cholestasis compared with a soybean oil-based lipid emulsion. The large sample size analyzed in our trial may be sufficient to verify a clinically meaningful effect. The preterm infants received intravenous lipid emulsion as early as $<24$ hours after birth. The trial recruited infants in a single center, thereby reducing possible bias by using one multidisciplinary team and a consistent protocol. Furthermore, we found that SMOFlipid provided a beneficial effect by decreasing the duration to full feeding days and preventing relevant complications such as ROP and BPD.

In the current literature the etiology of PN-associated cholestasis is multifactorial, with causes including prematurity, lack of enteral nutrition, sepsis, and factors relevant to the duration and composition of the PN [15-18]. Numerous adult studies have revealed that fish oil containing lipid emulsion has a positive effect on liver enzyme tests and prevention of PN-associated liver disease in surgical or critically ill patients [19-21]. Three studies resulted in a $15-25 \%$ faster plasma lipid clearance or $25-45 \%$ shorter half-life of the experimental fish oil containing lipid emulsion, even with different fish oil content [22-24]. Liang et al. showed that fish oil enriched PN produced a reduction in interleukin (IL) -6 , a high $\mathrm{CD} 4+/ \mathrm{CD} 8+$ ratio, and higher CD3+ and CD4+ lymphocytes in patients undergoing radical colorectal cancer resection [25]. These findings assumed that the supplement of EPA and DHA by the parenteral route may modulate immunocompetent cells in seriously ill surgical patients. Meanwhile, Socha et al. reported that infants with biliary atresia after surgery and intrahepatic cholestasis receiving long-chain PUFA supplemented formula had higher levels of docosahexaenoic acid (DHA) and arachidonic acid (ARA) [26]. Cholestasis status improved in the infants receiving long-chain PUFA supplementation. However, the number of cases was small, and fewer relevant studies were reported to support the speculation.

Retrospective animal research suggested that lipid emulsions containing a high concentration of $\omega-6$ LCPUFAs and phytosterols that produce proinflammatory prostaglandin E2 and thromboxane A2 mediated by the COX and 5-lipooxygenase pathways may induce immune response and promote hepatic injury [27]. In contrast, fish oil containing lipid emulsions rich in EPA and DHA that derive anti-inflammatory leukotriene B5, C5, D5, prostaglandin E3, prostagcyclin 13 , and thromboxane A3, also mediated by the COX and 5-lipooxygenase pathways, might reduce inflammation reaction and improve liver function. Besides, PN containing fish oil increased gene expression of canalicular bile acid transporters, resulted in greater secretion of hydrophobic bile acids and increased bile flow in neonatal piglets $[28,29]$ and rats [30]. These alterations may prevent cholestasis and explain the hepatoprotective effects of fish oil containing lipid emulsion. Pscheidl et al. also reported that a parenteral diet supplemented with fish oil normalized the nutritive blood flow to the gut, ameliorated the bactericidal defense of the splanchnic region and advanced killing of translocated bacteria in a low-dose endotoxin rat model [31]. We supposed that the result corresponded to decreased full feeding days in the SMOFlipid group in our trial. 
However, Repa et al.recently stated that SMOFlipid did not significantly reduce the incidence of PN associated cholestasis in extremely low birth weight infants [32]. In this study the intervention started within the first 120 hours of life, which was different from the intervention beginning within 24 hours of birth in our trial. On the other hand, the study was powered to detect a reduction of PN associated cholestasis from $25 \%$ to $10 \%$. The result showed that the incidence of PN associated cholestasis was $16 \%$ in the soybean oil-based lipid emulsion group, and $10 \%$ in the SMOFlipid group. The outcome of cholestasis was lower using SMOFlipid compared with soybean oil-based lipid emulsion, but the small population size and the low incidence of cholestasis limited the statistical significance of the differences.

The limitation of our study is that it is a retrospective cohort study, not a randomized control trial. Despite the fact that we used the same multidisciplinary team and a consistent protocol, there were some factors which could not be controlled. In addition, we did not quantitate the relative impact of DHA and AA levels in red blood cells to prove the direct correlation, and DHA and AA levels in breast milk to assess the influence of dietary intake. Further studies are needed a well-designed randomized control trial to evaluate the long-term effects of mixed lipid emulsion on growth, neurodevelopmental and visual outcomes in VLBW infants.

\section{Conclusion}

Parenteral nutrition of SMOFlipid was safe and well tolerated during the study period. Because of its relatively low $\omega-6: \omega-3$ ratio, SMOFlipid showed a potential beneficial influence on cholestasis and antiinflammatory effect in premature infants requiring PN. We also found that SMOFlipid was associated with a shorter duration of parenteral nutrition days and increased speed of enteral feeding. However, more randomized control trials are needed to prove the effect of different fish oil-based lipid emulsions in the prevention of $\mathrm{PN}$-associated cholestasis.

\section{Abbreviations}

PN: parenteral nutrition; LC-PUFAs: long-chain polyunsaturated fatty acids; MCTs: medium-chain triglycerides; ROP: retinopathy of prematurity; IVH: intraventricular hemorrhage; NEC: necrotizing enterocolitis; BPD: bronchopulmonary dysplasia; EPA: eicosapentaenoic acid; DHA: docosahexaenoic acid; AA: arachidonic acids

\section{Declarations}

\section{Acknowledgements}

The authors thank the staff at the Epidemiology and Biostatistics Center, Department of Changhua Christian Hospital for statistical assistance. The authors are grateful to the infants and their parents, as well as all the staffs in NICU of the Changhua Christian Children's Hospital for their assistance. 


\section{Authors' contributions}

YLW and $\mathrm{CCH}$ conceived and designed the study. YLW and LJC collected and analyzed the data. LYT, $\mathrm{HNC}, \mathrm{CHL}$ and $\mathrm{CCH}$ supervised data collection and analysis, reviewed and revised the manuscript. YLW and $\mathrm{CCH}$ finalized the manuscript. All authors read and approved the final manuscript as submitted and agree to be accountable for all aspects of the work and agree with its submission to BMC Pediatrics.

\section{Funding}

No funding received.

\section{Availability of data and materials}

The datasets generated and/or analysed during the current study are available to interested researchers from the corresponding author upon reasonable request.

\section{Ethics approval and consent to participate}

Ethical approval to conduct this study was obtained from the Institutional Review Board of Changhua Christian Hospital. Written consent from the caregivers of the neonates could not be obtained due to the retrospective nature of the study. However, all the patient-related information was anonymized.

\section{Consent for publication}

Not applicable.

\section{Competing interests}

The authors declare that they have no competing interests

\section{References}

1. Koletzko B, Goulet O, Hunt J, et al. Guidelines on Paediatric Parenteral Nutrition of the European Society of Paediatric Gastroenterology, Hepatology and Nutrition (ESPGHAN) and the European Society for ClinicalNutrition and Metabolism (ESPEN), Supported by the European Society of Paediatric Research (ESPR). J Pediatr Gastroenterol Nutr. 2005;41(Suppl 2):S1-87.

2. Tomsits E, Pataki M, Tölgyesi A, Fekete G, Rischak K, Szollár L. Safety and efficacy of a lipid emulsion containing a mixture of soybean oil, medium-chain triglycerides, olive oil, and 
fish oil: a randomised, double-blind clinical trial in premature infants requiring parenteral nutrition. $J$ Pediatr Gastroenterol Nutr. 2010 Oct;51(4):514-21.

3. Fallon EM, Le HD, Puder M. Prevention of parenteral nutrition-associated liver disease: role of omega-3 fish oil. Curr Opin Organ Transplant. 2010 Jun;15(3): 334-40.

4. Deshpande G, Simmer K, Deshmukh M, Mori TA, Croft KD, Kristensen J. Fish Oil (SMOFlipid) and olive oil lipid (Clinoleic) in very preterm neonates. J Pediatr Gastroenterol Nutr. 2014 Feb;58(2):177-82.

5. Rayyan M, Devlieger H, Jochum F, Allegaert K. Short-term use of parenteral nutrition with a lipid emulsion containing a mixture of soybean oil, olive oil, medium-chain triglycerides, and fish oil: a randomized double-blind study in preterm infants. JPEN J Parenter Enteral Nutr. 2012 Jan;36(1 Suppl):81S-94S.

6. Waitzberg DL, Torrinhas RS, Jacintho TM. New parenteral lipid emulsions for clinical use. JPEN J Parenter Enteral Nutr. 2006;30(4):351-67.

7. Krohn K, Koletzko B. Parenteral lipid emulsions in paediatrics. Curr Opin Clin Nutr Metab Care. 2006 May;9(3):319-23.

8. Wu GH, Zaniolo O, Schuster H, Schlotzer E, Pradelli L. Structured triglycerides versus physical mixtures of medium- and long-chain triglycerides for parenteral nutrition in surgical or critically ill adult patients: Systematic review and meta-analysis. Clin Nutr. 2017 Feb;36(1):150-161.

9. Sala-Vila A, Barbosa VM, Calder PC. Olive oil in parenteral nutrition. Curr Opin Clin Nutr Metab Care. 2007 Mar; 10(2):165-74.

10. Cai W, Calder PC, Cury-Boaventura MF, De Waele E, Jakubowski J, Zaloga G. Biological and Clinical Aspects of an Olive Oil-Based Lipid Emulsion-A Review. Nutrients. 2018 Jun 15;10(6). pii: E776.

11. Hsiao CC, Lin HC, Chang YJ, et al. Intravenous fish oil containing lipid emulsion attenuates inflammatory cytokines and the development of bronchopulmonary dysplasia in very premature infants: A double-blind, randomized controlled trial. Clin Nutr. 2018 Jun 18. pii: S02615614(18)31148-8.

12. Auestad N, Scott DT, Janowsky JS, et al. Visual, cognitive, and language assessments at 39 months: a follow-up study of children fed formulas containing long-chain polyunsaturated fatty acids to 1 year of age. Pediatrics. 2003 Sep;112(3 Pt 1):e177-83.

13. Innis SM. Dietary (n-3) fatty acids and brain development. J Nutr. 2007 Apr;137(4):855-9.

14. Neuringer M. Infant vision and retinal function in studies of dietary long-chain polyunsaturated fatty acids: methods, results, and implications. Am J Clin Nutr. 2000 Jan;71(1 Suppl):256S-67S.

15. Kelly DA. Liver complications of pediatric parenteral nutrition-epidemiology. Nutrition. 1998 Jan;14(1):153-7.

16. Andorsky DJ, Lund DP, Lillehei CW, et al. Nutritional and other postoperative management of neonates with short bowel syndrome correlates with clinical outcomes. J Pediatr. 2001 Jul;139(1):27-33. 
17. Alkharfy TM, Ba-Abbad R, Hadi A, Sobaih BH, AlFaleh KM. Total parenteral nutritionassociated cholestasis and risk factors in preterm infants. Saudi J Gastroenterol. 2014 SepOct;20(5):293-6.

18. Satrom K, Gourley G. Cholestasis in Preterm Infants. Clin Perinatol. 2016 Jun;43(2):355-73.

19. Ma CJ, Wu JM, Tsai HL, et al. Prospective double-blind randomized study on the efficacy and safety of an $\mathrm{n}-3$ fatty acid enriched intravenous fat emulsion in postsurgical gastric and colorectal cancer patients. Nutr J. 2015 Jan 21;14:9.

20. Manzanares W, Langlois PL, Dhaliwal R, Lemieux M, Heyland DK. Intravenous fish oil lipid emulsions in critically ill patients: an updated systematic review and meta-analysis. Crit Care. 2015 Apr 16;19:167.

21. Mateu de Antonio J, Florit-Sureda M. Effects unrelated to anti-inflammation of lipid emulsions containing fish oil in parenteral nutrition for adult patients. Nutr Hosp. 2017 Feb 1;34(1):193-203.

22. Simoens CM, Deckelbaum RJ, Massaut JJ, Carpentier YA. Inclusion of $10 \%$ fish oil in mixed mediumchain triacylglycerol-long-chain triacylglycerol emulsions increases plasma triacylglycerol clearance and induces rapid eicosapentaenoic acid (20:5n-3) incorporation into blood cell phospholipids. Am J Clin Nutr. 2008 Aug;88(2):282-8.

23. Carpentier YA, Hacquebard M, Portois L, Dupont IE, Deckelbaum RJ, Malaisse WJ. Rapid cellular enrichment of eicosapentaenoate after a single intravenous injection of a novel medium-chain triacylglycerol:fish-oil emulsion in humans. Am J Clin Nutr. 2010 Apr;91(4):875-82.

24. Schlotzer E, Kanning U. Elimination and tolerance of a new parenteral lipid emulsion (SMOF): $A$ double-blind cross-over study in healthy male volunteers. Ann Nutr Metab. 2004;48(4):263-8.

25. Liang B, Wang S, Ye YJ, et al. Impact of postoperative omega-3 fatty acid-supplemented parenteral nutrition on clinical outcomes and immunomodulations in colorectal cancer patients. World $\mathrm{J}$ Gastroenterol. 2008 Apr 21;14(15):2434-9.

26. Socha P, Koletzko B, Jankowska I, et al. Long-chain PUFA supplementation improves PUFA profile in infants with cholestasis. Lipids. 2002 Oct;37(10):953-7.

27. Le HD, Meisel JA, de Meijer VE, Gura KM, Puder M. The essentiality of arachidonic acid and docosahexaenoic acid. Prostaglandins Leukot Essent Fatty Acids. 2009 Aug-Sep;81(2-3):165-70.

28. Van Aerde JE, Duerksen DR, Gramlich L, et al. Intravenous fish oil emulsion attenuates total parenteral nutrition-induced cholestasis in newborn piglets. Pediatr Res. 1999 Feb;45(2):202-8.

29. Lavallee CM, Lim DW, Wizzard PR, et al. Impact of Clinical Use of Parenteral Lipid Emulsions on Bile Acid Metabolism and Composition in Neonatal Piglets. JPEN J Parenter Enteral Nutr. 2018 Aug 23.

30. Chen WJ, Yeh SL. Effects of fish oil in parenteral nutrition. Nutrition. 2003 Mar;19(3):275-9.

31. Pscheidl E, Schywalsky M, Tschaikowsky K, Böke-Pröls T. Fish oil-supplemented parenteral diets normalize splanchnic blood flow and improve killing of translocated bacteria in a low-dose endotoxin rat model. Crit Care Med. 2000 May;28(5):1489-96. 
32. Repa A, Binder C, Thanhaeuser M, et al. A Mixed Lipid Emulsion for Prevention of Parenteral Nutrition Associated Cholestasis in Extremely Low Birth Weight Infants: A Randomized Clinical Trial. J Pediatr. 2018 Mar;194:87-93.e1.

\section{Tables}

Table 1. Demographic characteristics

\begin{tabular}{|l|l|l|l|}
\hline Perinatal data & $\begin{array}{l}\text { SMOFlipid }(\mathrm{N}= \\
195)\end{array}$ & $\begin{array}{l}\text { Lipovenoes }(\mathrm{N}= \\
204)\end{array}$ & $\begin{array}{l}P \\
\text { value }\end{array}$ \\
\hline Gestational age (weeks), mean $\pm \mathrm{SD}$ & $28.93 \pm 2.51$ & $28.84 \pm 2.53$ & 0.723 \\
\hline Birth body weight (g), mean \pm SD & $1122.88 \pm 294.56$ & $1111.51 \pm 286.87$ & 0.696 \\
\hline Birth height (cm), mean \pm SD & $36.5 \pm 3.8$ & $36.4 \pm 3.5$ & 0.665 \\
\hline $\begin{array}{l}\text { Birth head circumference (cm), mean } \\
\pm \text { SD }\end{array}$ & $26.2 \pm 2.8$ & $25.8 \pm 2.5$ & 0.095 \\
\hline Apgar score (1), medium \pm SD & $7 \pm 1.74$ & $6 \pm 1.62$ & 0.357 \\
\hline Apgar score (5), medium \pm SD & $8 \pm 1.21$ & $8 \pm 1.25$ & 0.220 \\
\hline $\begin{array}{l}\text { Duration of parenteral nutrition } \\
\text { (days), }\end{array}$ & $18 \pm 9.31$ & $25.5 \pm 14.36$ & $<$ \\
\hline medium \pm SD & & & 0.001 \\
\hline $\begin{array}{l}\text { Duration to full enteral feeding (days), } \\
\text { medium } \pm \text { SD }\end{array}$ & $25 \pm 10.33$ & $33 \pm 16.22$ & $<$ \\
\hline Breast milk, n (\%) & & & 0.001 \\
\hline Formula milk, n (\%) & $109(55.9)$ & $124(62)$ & 0.083 \\
\hline Mixed breast and formula milk, $\mathrm{n}(\%)$ & $46(23.6)$ & $35(17.5)$ & 0.104 \\
\hline $\begin{array}{l}\text { Hospitalization day (days), medium } \pm \\
\text { SD }\end{array}$ & $54 \pm 26.49$ & $58 \pm 27.93$ & 0.245 \\
\hline
\end{tabular}

Table 2. Primary outcome and the relevant clinical laboratory 


\begin{tabular}{|c|l|l|l|}
\hline & $\begin{array}{l}\text { SMOFlipid }(\mathrm{N}= \\
195)\end{array}$ & $\begin{array}{l}\text { Lipovenoes }(\mathrm{N}= \\
204)\end{array}$ & P value \\
\hline Cholestasis, $\mathrm{n}(\%)$ & $20(10.3)$ & $41(20.1)$ & 0.006 \\
\hline Hematological parameters & & & \\
\hline WBC $(/ \mu \mathrm{L})$, mean $\pm \mathrm{SD}$ & $15807.18 \pm 6947.41$ & $16516.67 \pm 6519.84$ & 0.718 \\
\hline Hb $(\mathrm{g} / \mathrm{dL})$, mean $\pm \mathrm{SD}$ & $10.34 \pm 1.43$ & $10.07 \pm 1.35$ & 0.047 \\
\hline Hct $(\%)$, mean $\pm \mathrm{SD}$ & $30.51 \pm 4.22$ & $29.5 \pm 3.73$ & 0.011 \\
\hline Platelet $(103 / \mu \mathrm{L})$, mean $\pm \mathrm{SD}$ & $382.14 \pm 186.49$ & $392.61 \pm 152.95$ & 0.541 \\
\hline Biochemical parameters & & & \\
\hline CRP $(\mathrm{mg} / \mathrm{dL}), \mathrm{mean} \pm \mathrm{SD}$ & $1.01 \pm 1.58$ & $0.82 \pm 1.81$ & 0.22 \\
\hline$\gamma$-GT $(\mathrm{U} / \mathrm{L}), \mathrm{mean} \pm \mathrm{SD}$ & $107.57 \pm 92.67$ & $131.12 \pm 98.79$ & 0.019 \\
\hline $\begin{array}{l}\text { Triglyceride }(\mathrm{mg} / \mathrm{dL}), \mathrm{mean} \pm \\
\text { SD }\end{array}$ & $127.91 \pm 70.14$ & $186.78 \pm 165.27$ & $<$ \\
\hline $\begin{array}{l}\text { Cholesterol }(\mathrm{mg} / \mathrm{dL}), \text { mean } \pm \\
\text { SD }\end{array}$ & $150.36 \pm 33.92$ & $158.39 \pm 35.9$ & 0.023 \\
\hline
\end{tabular}

Table 3. Multivariable analysis on the risk for PN-associated cholestasis

\begin{tabular}{|l|l|l|l|}
\hline Parameters & Odds ratio & $95 \%$ CI & $P$ value \\
\hline SMOFlipid & 0.397 & $0.216-0.730$ & 0.003 \\
\hline GPT & 1.012 & $1.001-1.022$ & 0.024 \\
\hline Hct & 0.964 & $0.947-0.982$ & $<0.001$ \\
\hline Albumin & 0.353 & $0.163-0.767$ & 0.008 \\
\hline Glucose & 0.973 & $0.954-0.992$ & 0.005 \\
\hline
\end{tabular}


Table 4. Secondary outcomes

\begin{tabular}{|l|l|l|l|}
\hline & SMOFlipid $(\mathrm{N}=195)$ & Lipovenoes $(\mathrm{N}=204)$ & $P$ value \\
\hline ROP, stage 3, n (\%) & $7(3.6)$ & $29(14.3)$ & $<0.001$ \\
\hline IVH, grade 3,4, n (\%) & $8(4.1)$ & $5(2.5)$ & 0.235 \\
\hline NEC, n (\%) & $11(5.6)$ & $15(7.4)$ & 0.489 \\
\hline BPD, n (\%) & $72(36.9)$ & $95(46.7)$ & 0.046 \\
\hline Sepsis, n (\%) & $44(22.5)$ & $52(25.5)$ & 0.543 \\
\hline Mortality, n (\%) & $2(1.0)$ & $7(3.4)$ & 0.176 \\
\hline
\end{tabular}

